\title{
Retroviral Glycoprotein-Mediated Immune Suppression via the potassium Channel KCa3.1- A new strategy for treatment of Inflammatory Bowel Diseases.
}

\author{
Magdalena Laska ${ }^{1}$, Jesper Bonnet Møller², Jonas Heilskov Graversen², Dorte Strøbæk ${ }^{3}$, \\ Linda Blomster ${ }^{3}$, Palle Christophersen ${ }^{3}$, and Shervin Bahrami ${ }^{1}$ \\ ${ }^{1}$ Aarhus University \\ ${ }^{2}$ University of Southern Denmark \\ ${ }^{3}$ Saniona A/S
}

January 10, 2022

\begin{abstract}
Background and Purpose: Peptides derived from retroviral envelope proteins have been shown to possess a wide range of immunosuppressive and anti-inflammatory activities. We have previously reported identification of such a peptide derived from the envelope protein coded by a human endogenous retrovirus (HERV). In this study we assessed effects of this peptide treatment on inhibition of immune response in the DSS-induced mice model of colitis. Furthermore, we identified that in vitro the peptide inhibits the KCa3.1 potassium channel, a potential target for therapy of immune diseases. Experimental Approach: We characterized an immunosuppressive peptide ENV59, from a specific HERV envelope protein, in vivo effects on inflammation control in acute colitis mice model and in vitro on the production of pro-inflammatory cytokines. Furthermore, we described in vitro ENV59-GP3 effects with respect to potency of inhibition on KCa3.1 channels and calcium influx. Key Results: ENV59-GP3 peptide treatment showed reduction of the disease score in the DSS-induced acute colitis mice model, which was comparable to effects of the KCa3.1 channel blocker NS6180. Analysis of cytokine production from DSS-mice model treated animals revealed equipotent inhibitory effects of the ENV59-GP3 and NS6180 compounds on the production of IL-6, TNF- $\alpha$, IL-1 $\beta$. Patch clamp studies show that the peptide ENV59-GP3 is a blocker of the potassium channel KCa3.1. Conclusion and Implications: Env59-GP3 represents KCa3.1 channel inhibitor underlining the implications of using virus derived channel blockers for treatment of autoimmune diseases. There are no drugs with a similar mechanism of action currently on the market.
\end{abstract}

\section{Introduction}

Inflammatory Bowel Disease (IBD) is a group of inflammatory conditions of the colon and small intestine. Crohn's disease (CD) and ulcerative colitis (UC) are the principal types of inflammatory bowel diseases. The etiology is multifactorial, and many genetic loci have been found to serve as susceptibility genes for different subgroups of patients with IBD [1,2], likewise, environmental influences are also considered as factors involved in IBD onset [3]. In addition, to its complicated risk factors, the pathogenesis of IBD is not entirely clear, making it difficult to develop new drugs with therapeutic value and acceptable side effects [2].

For more than two decades, animal models of intestinal inflammation have provided a wealth of information about mucosal immunology and the intestinal inflammation encountered in the inflammatory bowel diseases. While no single model has proven to sufficiently represent the complexity of the clinical characteristics of the human disease, the combined data obtained from these various animal models have provided a more detailed understanding of the underlying principles of human IBD and delineate pathways that might help predict the successful outcome of novel therapeutics in the clinic $[4,5]$. 
The most widely used murine model of colitis employs dextran sodium sulfate (DSS), a chemical colitogen with anticoagulant properties, to induce intestinal epithelial damage [6]. The DSS colitis model lends itself to IBD research due to its rapidity, simplicity, reproducibility and controllability. Acute, chronic and relapsing models of intestinal inflammation can be achieved by modifying the concentration of DSS and the frequency of administration [6].

Numerous inflammatory mediators have been implicated in the pathogenesis of IBD. These include cytokines, eicosanoids, reactive oxygen species, nitric oxide, and complement activation products [7]. Similarly, increased expression of different inflammatory mediators (TNF- $\alpha$, IL-1 $\beta$, IFN-g, IL-10 and IL-12) are observed as early as the first day of DSS treatment. The production of these inflammatory mediators increases progressively during DSS treatment [8].

Recently, we reported identification of an immunosuppressive peptide (ENV59-GP3) from a specific human endogenous retroviral envelope protein, ENV59, which has been adapted by the immune system and is involved in ameliorating autoimmunity $[9,10]$. The ENV59-GP3 peptide showed strong immune modulating activity both in vitro andin vivo in SKG mouse model for rheumatoid arthritis and EAE model for Multiple Sclerosis $[9,10]$.

In this study we follow up on our previous findings and demonstrate that the ENV59-GP3 peptide treatment, reduces inflammation in the DSS-induced mice model of colitis. Furthermore, we show that in vitro ENV59GP3 inhibits the $\mathrm{K}_{\mathrm{Ca}} 3.1$ potassium channel, an ion channel responsible for hyperpolarization of the plasma membrane and regulation of calcium influx upon lymphocyte activation.

Potassium channels might be regarded as a new group of therapeutic targets for IBD [11, 12]. The intermediate-conductance calcium/calmodulin-gated potassium channel $\mathrm{K}_{\mathrm{Ca}} 3.1$ also known as the IK or the Gardos channel (encoded by the $K C N N_{4}$ gene) is expressed in a variety of tissues and cell types including T cells [13, 14], macrophages as well as DCs [14]. In T cells, sustained $\mathrm{Ca}^{2+}$ influx via the CRAC channel is important for sufficient cytokine production and proliferation. Influx of $\mathrm{Ca}^{2+}$ is dependent on a negative membrane potential. Activation of $\mathrm{K}_{\mathrm{Ca}} 3.1$ by elevated intracellular $\mathrm{Ca}^{2+}$ maintains the negative membrane potential through efflux of $\mathrm{K}^{+}$, which helps to sustain $\mathrm{Ca}^{2+}$ entry into the cell. Upon channel opening, the efflux of potassium is driven by its concentration gradient and results in hyperpolarization of the membrane providing the electrochemical gradient necessary for influx of calcium [15].

$\mathrm{K}_{\mathrm{Ca}} 3.1$ channel is known to be involved in regulating $\mathrm{T}$ cell function, proliferation, and cytokine production [16], thus suggesting that they could be involved in the pro-inflammatory responses in IBD further up-stream of inflammatory cytokines such as IFN-g and TNF- $\alpha$. Furthermore, studies have revealed a prominent role of KCNN4 in $\mathrm{Ca}^{2+}$-dependent ion transport and intestinal restitution [17]. In a $\mathrm{T}$ cell-mediated mouse model of colitis, inhibition of $\mathrm{K}_{\mathrm{Ca}} 3.1$ ameliorated the disease [11]. Likewise, the $\mathrm{K}_{\mathrm{Ca}} 3.1$ channel blocker NS6180 prevents T-cell activation and inflammation in a rat model of inflammatory bowel disease [18]. Although loss of $\mathrm{K}_{\mathrm{Ca}} 3.1$ did not interfere with $\mathrm{CD} 4 \mathrm{~T}$ cell differentiation, both $\mathrm{Ca}^{2+}$ influx and cytokine production were impaired in KCNN4-/- Th1 and Th2 CD4 T cells, whereas T-regulatory and Th17 function were normal. In animal models for other autoimmune diseases, the $\mathrm{K}_{\mathrm{Ca}} 3.1$ inhibitor TRAM-34 or ICA-17043 (Senicapoc) have been reported to prevent experimental autoimmune encephalomyelitis in mice [19] or to inhibit/attenuate inflammatory responses in mice with collagen antibody induced rheumatoid arthritis. Clinical studies with Clotrimazole (form which Tram-34 has been derived) already provided evidence that blockers of this channel may be useful in treatment of chronic diseases such as RA, however, non-target related adverse effects have prevented further development in some cases [20].

\section{Materials and methods}

Preparation of a synthetic peptide

ENV59-GP3 (AJ289711.1 envelope sequence) peptide and Flu ISU (control) peptide were chemically synthesized and acquired from Schaefer-N, Copenhagen, DK with. All peptides were homo-dimerized through 
disulfide bond formation between cysteine residues. The dimeric forms were used in all subsequent experiments.

\section{Cell culture}

THP-1 human acute monocytic leukemia cells and HEK 293 cells were grown in culture in Roswell Park Memorial Institute medium (RPMI 1640, Invitrogen) or DMEM (Dulbecco's Modified Eagle Medium) respectively, supplemented with 10\% fetal bovine serum (Sigma, Cat\# F7524) and 1\% penicillin/streptomycin $(\mathrm{P} / \mathrm{S})$.

(qRT-PCR) analysis

Quantitative reverse transcription-polymerase chain reaction (qRT-PCR) analysis were performed as described previously using primers for amplification of IL-6 and TNF-alpha [9, 21].

Enzyme-linked immunosorbent assay (ELISA)

IL-6 or TNF-alpha was assayed with a conventional sandwich ELISA (BioLegend), using ELISA (BioLegend), using 96-well plates coated with antibodies specific for IL-6 or TNF-alpha.

Mouse model of DSS-induced colitis

In vivo studies were conducted at the University of Southern Denmark using male 8-10 weeks old C57BI/6n littermates (Taconic). The mice were co-housed in groups of 4 and provided with water and food ad libitum at $20-24^{\circ} \mathrm{C}, 55 \%$ humidity and a 12 -h light/dark cycle. All procedures were performed in accordance with the ethical standards of the institution with approval from the Danish Animal Experiments Inspectorate (Approval no. 2017-15-0201-01254).

DSS-induced acute intestinal inflammation

For acute DSS-induced colitis, the mice were administrated 2.5\% DSS (molecular weight, 36-50kD; MP Biomedicals) in their drinking water for 7 days, followed by ordinary tap water. Three treatment groups were included in the study: animals treated with $12,5 \mu \mathrm{g}$ peptide $(250 \mu \mathrm{l})$ IP every other day $(0.5 \mathrm{mg} / \mathrm{kg})$; animals treated with saline $(250 \mu \mathrm{l})$ as control; or animals treated with $62,5 \mu \mathrm{g}$ blocker $(250 \mu \mathrm{l})$ IP every other day $(2.5 \mathrm{mg} / \mathrm{kg})$. All buffers contain $1.25 \%$ DMSO. During this time period, the mice were weighted daily and subsequently sacrificed at day 10. At the time of sacrifice, colon specimens were collected for histology and $\mathrm{qPCR}$ analyses.

\section{Histological assessment of inflammation}

Assessment of inflammation was performed as described by [22]. In brief, colonic sections were H\&E-stained and assessed for disease severity by trained personnel blinded to treatment and genotype. Scoring was based on two parameters; inflammatory cell infiltrate (0-3) and intestinal architecture (0-3). The combined values were added to give a histological score between 0 and 6 . Images were acquired using an inverted Nikon Eclipse Ti microscope.

\section{Q-PCR analyses for mouse cytokines}

For transcriptional analyses, colon samples from DSS-treated mice were homogenized in TRIzol (Thermo Fisher Scientific) and stored at $-80^{\circ} \mathrm{C}$ until further processed. RNA was initially extracted with chloroform and then further purified by lithium chloride precipitation to remove DSS a strong inhibitor of downstream PCR applications [23]. Reverse transcription of total RNA was performed using the High-Capacity cDNA Reverse Transcription kit according to the manufacturer's recommendations (Thermo Fisher Scientific). Quantification of expression was detected on a StepOnePlus Real-Time PCR (Thermo Fisher Scientific) using the following TaqMan Gene Expression Assays (Applied Biosystems):Il-1b (Mm00434228), Il-6 (Mm00446190), $I L-17 a(\mathrm{Mm00439618)}$ and tnfa (Mm00443258). Gene expression was normalized as n-fold difference to mouse Hprt1 (Mm00446968) according to the cycling threshold.

Electrophysiology 
All experiments were performed in the whole-cell version of the patch-clamp technique using either an EPC-9 or EPC-10 amplifier controlled by Pulse software (HEKA Electronics, Lambrecht, Germany) according to the previously described methodology [18]. HEK293 cells stably expressing human wild-type $\mathrm{K}_{\mathrm{Ca}} 3.1$ channel or THP-1 human monocyte cell line were used. $\mathrm{IC}_{50}$ values for blocking $\mathrm{K}_{\mathrm{Ca}} 3.1$ channels were estimated either from concentration-response curves by fitting to the Hill equation or from a fit to the inhibition kinetics assuming that $\mathrm{IC}_{50}=K_{\mathrm{d}}$ as detailed previously [18]. Selectivity experiments using other ion channels were performed as described previously [24].

Calcium influx assay

Intracellular calcium measurements in cell populations were monitored by using Fluo-8 calcium flux assay according to the manufactures protocol (Abcam, Denmark) and fluorescence was analyzed at at Ex/Em 490/525 nm. Depletion of intracellular stores was achieved by addition of 250nM Thapsigargin (Tg; Sigma).

\section{Results}

Effect of the ENV59-GP3 peptide and $\mathrm{K}_{\mathrm{Ca}} 3.1$-blocker NS6180 in DSS-induced acute colitis mice model

The effect of blocking $\mathrm{K}_{\mathrm{Ca}} 3.1$ on the immune responsein vivo was evaluated using DSS-induced acute colitis model. Starting at day 0 and throughout the course of the experiment, the mice were dosed i.p. twice weekly with a ENV59-GP3 peptide, at $0.5 \mathrm{mg}$ per $\mathrm{kg}$ of body weight; selective $\mathrm{K}_{\mathrm{Ca}} 3.1$ blocker NS6180 at $2.5 \mathrm{mg}$ per $\mathrm{kg}$ of body weight, or with saline (control). The development of IBD was monitored as daily average weight loss development. As shown in Figure 1A, DSS administration resulted in significant weight loss (control), whereas in mice treated with ENV59-GP3 peptide or the channel blocker NS6180, weight loss was significantly reduced by day $6 \sim 10$. Furthermore, we found that colon length, a macroscopic indicator of colitis severity, in DSS-treated mice control group was significantly reduced. In contrast, administration of ENV59-GP3 at $0.5 \mathrm{mg} / \mathrm{kg}$ or NS6180 at $2.5 \mathrm{mg} / \mathrm{kg}$ led to a marked improvement in colon length $(p$ $<0.0001$ ) (Figure 1B). Additionally, we tested whether treatments with ENV59-GP3 peptide or NS6180 blocker would have an impact on expression of proinflammatory cytokines in colon tissues, presumably as an effect of upstream blockage of the $\mathrm{K}_{\mathrm{Ca}} 3.1$ channel. Consistent with less severe disease, TNF- $\alpha$, IL-1 $\beta$ and IL-6 (Figure 1C) show decreased expression in ENV59-GP3 and NS6180 treated animals, though only difference in IL-6 expression was statistically significant. Unfortunately, such a high degree of variability in RT-qPCR analyses of inflammatory mediators are often observed in similar in vivo models. Furthermore, the expression of IL-17 mRNA was not markedly reduced by either tested treatments. At the time of sacrifice, ENV59-GP3 and/or NS6180 treated mice displayed significantly reduced colonic shortening and decreased intestinal inflammation compared with control group (Figure 2).

\section{ENV59-GP3 Immune Suppression via Inhibition of the Intermediate Conductance Calcium} Activated Potassium Channel $\mathrm{K}_{\mathrm{Ca}} 3.1$

Despite the extensive literature documenting a broad range of immune suppressive activity affecting different signal transduction pathways by retroviral derived immune suppressive peptides, no precise molecular mechanism of action (MoA) has been described. ENV59-GP3 is derived from an endogenous retroviral envelope protein and thus is expressed on the surface of immune cells. Therefore, it can be expected that it will interact with a surface receptor. We noted that $\mathrm{K}_{\mathrm{Ca}} 3.1$ is a surface protein and that inhibition of $\mathrm{K}_{\mathrm{Ca}} 3.1$ protected mice from developing severe colitis in two mouse models of inflammatory bowel disease [11], furthermore $\mathrm{K}_{\mathrm{Ca}} 3.1$ inhibitor ameliorate the disease progression in EAE mice [19]. The overlap between the activity of $\mathrm{K}_{\mathrm{Ca}} 3.1$ and our observations of the ENV59 activity made it a good candidate for further investigation.

\section{Cytokine release and inhibiting effect of ENV59-GP3 and of NS6180 in stimulated THP-1 cells}

The ENV59-GP3 peptide inhibits the production of pro-inflammatory cytokines in activated THP-1 cells [9]. To elucidate whether $\mathrm{K}_{\mathrm{Ca}} 3.1$ plays any role in this phenotypic activity of ENV59-GP3 peptide, expression and activity of the $\mathrm{K}_{\mathrm{Ca}} 3.1$ channels in THP-1 cells were confirmed by qPCR (Figure 3B). Patch clamp experiments confirmed existence of currents sensitive to specific $\mathrm{K}_{\mathrm{Ca}} 3.1$ channel blocker (NS6180) and channel 
opener (NS309, Figure 3A). Next we studied if the inhibitory effect of ENV59-GP3 in cytokine production mimics that of NS6180, a potent and highly selective small molecule inhibitor of $\mathrm{K}_{\mathrm{Ca}} 3.1$ channels [18]. Our results (Figure $3 \mathrm{C}$ ) corroborate the notion that $\mathrm{K}_{\mathrm{Ca}} 3.1$ is an important regulator of synthesis and release of proinflammatory cytokines in THP-1 cells and that the ENV59-GP3 peptide and the channel blocker NS6180 have similar effects on cytokine production in these cells.

\section{Effect of ENV59-GP3 peptide on calcium influx following $\mathrm{Ca}^{2+}$ depletion in THP-1 cells}

To measure the effects of ENV59-GP3 peptide and NS6180 blocker on calcium flux, THP-1 cells were loaded with the calcium sensitive dye Fluo-4 and incubated with either ENV59-GP3 peptide or NS6180 blocker, and/or with the calcium store depleting agent Thapsigargin, an inhibitor of the sarco/endoplasmic reticulum $\mathrm{Ca}^{2+}$ ATPase (SERCA) [25] (Figure 4A). Similarly, both ENV59-GP3 peptide and NS6180 reduced the influx-mediated $\mathrm{Ca}^{2+}$ accumulation induced by Thapsigargin. In comparison, an immune suppressive peptide isolated from the influenza hemagglutinin (Flu ISU) which has a similar immunosuppressive phenotype to ENV59-GP3 [21], but interacts with and inhibits dimerization of the stimulator of interferon genes (STING) [21] did not have a significant effect in the $\left[\mathrm{Ca}^{2+}\right]_{\mathrm{I}}$ in this assay. Thus, the ENV59-GP3 peptide's biological activity is more similar to the $\mathrm{K}_{\mathrm{Ca}} 3.1$ inhibitor than it is to an influenza virus-derived immunosuppressive peptide.

\section{Selective inhibition of $\mathrm{K}_{\mathrm{Ca}} 3.1$ channels in THP-1 cells by ENV59-GP3}

We next examined the direct effect of the ENV59-GP3 peptide on the essentially pure $\mathrm{K}_{\mathrm{Ca}} 3.1$ current (Figure 4B-C) at $-40 \mathrm{mV}$ in THP-1 cells (Figure 4B). ENV59-GP3 peptide was applied at concentrations relevant for its immunosuppressive effects (Figure 3C) and, as clearly shown, it reversibly inhibited the $40 \mathrm{mV}$ current component, which was formally identified as being $\mathrm{K}_{\mathrm{Ca}} 3.1$ by the subsequent application of NS6180. Furthermore, the ENV59-GP3 peptide-like NS6180-did not exert a measurable effect on the voltage dependent current component at positive potentials. Hence the effect seems selective for the $\mathrm{K}_{\mathrm{Ca}} 3.1$ channel over $\mathrm{K}_{\mathrm{v}}$ channels in THP-1 cells.

ENV59-GP3 inhibition of recombinant human $K_{\mathrm{Ca}} 3.1$ channels stably expressed in HEK293 cells

Figure 4D-E summarizes the results for ENV59-GP3 with the curve representing fits to the Hill equation, revealing $\mathrm{IC}_{50}$ value of $43 \mu \mathrm{M}$ for ENV59-GP3. To confirm that the inhibiting effect of ENV59-GP3 peptide represent an interaction with the $\mathrm{K}_{\mathrm{Ca}} 3.1$ channel and not an indirect effect, we evaluated the peptide's effect on the recombinant $\mathrm{hK}_{\mathrm{Ca}} 3.1$ stably over-expressed in HEK293 cells (Figure $5 \mathrm{~A}-\mathrm{D}$ ), a cell type which does not naturally express any calcium activated potassium channels. As observed in the previous experiment with THP-1 cells, after the initial transient rise in activity, the $\mathrm{K}_{\mathrm{Ca}} 3.1$ current stabilized. Initially we blocked the current by using Charybdotoxin-a very potent $\mathrm{K}_{\mathrm{Ca}} 3.1$ blocker [26]. The ENV59-GP3 peptide exert a qualitatively similar reversible inhibition, whereas the control peptide Flu ISU did not show any effect, in accordance with its lack of effect in the $\left[\mathrm{Ca}^{2+}\right]$ assay (Figure $5 \mathrm{C}-\mathrm{D}$ ). Finally, the NS309 activator was applied yielding the expected specific increase in $\mathrm{K}_{\mathrm{Ca}} 3.1$ current as also observed for the THP-1 channel.

\section{Discussion}

The ENV59-GP3 peptide contains the ISD sequence from a specific human endogenous retroviral protein encoded by the envelope ENV59 gene, originally identified in SLE patients [9], and shown to be adapted by the human immune system to ameliorate autoimmunity $[9,10]$. In this report, we provide evidence that treatment with the ISD ENV59-GP3 inhibits the development of colitis in dextran sulfate sodium (DSS) -induced mice, a widely recognized autoimmune model of human IBD. ENV59-GP3 showed reduction of the disease score in this model, which was comparable to effects of the $\mathrm{K}_{\mathrm{Ca}} 3.1$ channel blocker NS6180, though ENV59-GP3 treatment was at five times lower doses (by weight) than that of NS6180. The very low doses of ENV59-GP3 in in vivo treatment regime are in agreement with the treatment results in other animal models $[9,10]$.

In our literature review for potential signaling pathways and molecular targets utilized by ENV59-GP3 
peptide we came across studies by Di et al. who has reported that that $\mathrm{K}_{\mathrm{Ca}} 3.1$ ( KCNN4 ) - / - mice are protected from developing severe colitis [11]. Similarly, as is the case for ENV59-GP3, NS6180 or Tram-34small molecule inhibitors of $\mathrm{K}_{\mathrm{Ca}} 3.1$ reduce the disease score in T-cell mediated colitis models [11].

This prompted us to study the effects of Env59-GP3 peptide on the calcium activated potassium channels. Here, for the first time, we show the inhibitory activity of an endogenous retroviral ISD peptide on the intermediate-conductance calcium activated potassium-ion channel $\mathrm{K}_{\mathrm{Ca}} 3.1$ current.

$\mathrm{K}_{\mathrm{Ca}} 3.1$ plays an important function in regulating $\mathrm{Ca}^{2+}$ influx - mediated functions such as cytokine production, proliferation and migration in the cells of the immune system [27]. In line with this mechanism, T-cell receptor engagement dramatically induces $\mathrm{K}_{\mathrm{Ca}} 3.1$ expression, in parallel with increased $\mathrm{K}_{\mathrm{Ca}} 3.1$ currents and enhanced $\left[\mathrm{Ca}^{2+}\right]$ signaling during human T-cell activation $[12,27] . \mathrm{K}_{\mathrm{Ca}} 3.1$ has been recognized as a potent target for several autoimmune diseases indications including IBD, atherosclerosis, multiple sclerosis, rheumatoid arthritis as well as diseases such as asthma, renal fibrosis, diabetes and sickle cell anemia [15, 28, 29].

Analysis of cytokine production revealed similar inhibitory effects of the ISD ENV59-GP3 peptide and the selective $\mathrm{K}_{\mathrm{Ca}} 3.1$ blocker NS6180 on the in vitro production of IL-6 and TNF-alpha in the LPS or PMA + ionomycin PBMCs (data not shown) assays reflecting a particularly important role of $\mathrm{K}_{\mathrm{Ca}} 3.1$ channel when proliferation is driven by the $\mathrm{Ca}^{2+}$-dependent NFAT and NF- $k$ B pathways. The identical cytokine profiles obtained with ENV59-GP3 and $\mathrm{K}_{\mathrm{Ca}} 3.1$ inhibitors strongly support their identical pharmacological activity in these assays. Analysis of cytokine production from DSS-mice model treated animals revealed equipotent inhibitory effects of the ENV59-GP3 and NS6180 compounds on the production of IL-6, TNF- $\alpha$, IL-1 $\beta$ - whereas IL-17 was not affected by the peptide and only minorly affected by the NS6180 treatment. These findings also reinforce a critical role for Th1 effector cells in murine model of colitis, and are consistent with a recent report demonstrating that IL-17 may have more of a protective function by interfering with the generation of Th1 cells [30]. Thus, by not inhibiting Treg and Th17 cell function, $\mathrm{K}_{\mathrm{Ca}} 3.1$ blockers may have a unique therapeutic profile to inhibit procolitis-inducing Th1 and Th2 cells, without interfering with the beneficial function of Treg and possibly Th17 cells [10].

Env59-GP3 peptide inhibits calcium influx induced by Thapsigargin in a variety of different cell types. Thapsigargin is an inhibitor of the sarco/endoplasmic reticulum $\mathrm{Ca}^{2+}$ ATPase (SERCA). Inhibition of SERCA causes a slight increase of intracellular $\mathrm{Ca} 2+$, which in turn activates the calcium release-activated channels (CRAC), resulting in significant influx of calcium ions into the cytoplasm. This calcium influx is inhibited by ENV59-GP3. Interestingly, ENV59-GP3 showed no direct effect on the calcium release-activated channels (CRAC) itself (data not shown), in agreement with the peptide's inhibitory effect on the calcium activate potassium efflux through $\mathrm{K}_{\mathrm{Ca}} 3.1$.

The immune suppressive effect of ENV59-GP3 on THP-1 cells described in this report can be accredited to its inhibitory effect on the ion channel, since electrophysiological recordings as well as mRNA expression levels demonstrated the presence and the activity of $\mathrm{K}_{\mathrm{Ca}} 3.1$ in this human monocyte cell line.

$\mathrm{K}_{\mathrm{Ca}} 3.1$ expression has been correlated with IBD in clinical settings. The impact of KCNN4 expression or activity was extensively analyzed in immune cells. T cell activation is altered by the expression level and activity of KCNN4 in T lymphocytes in pediatric patients with IBD [31]. The expression level of KCNN4 in $\mathrm{T}$ cells can be correlated with disease activity in UC and elevated T-cell KCNN4 expression in UC correlates with inflammatory cytokine levels and disease activity [26]. In CD a gene variant of $K C N N_{4}$ has been described that is associated with ileal disease in the New Zealand and Australian population [32].

In addition to IBD, $\mathrm{K}_{\mathrm{Ca}} 3.1$ channels are suggested to be a novel target for treating various diseases such as idiopathic pulmonary fibrosis [33], airway inflammation (allergic asthma) and ischemia/stroke [34]. Senicapoc, a $\mathrm{K}_{\mathrm{Ca}} 3.1$ blocker, was suggested as a treatment for sickle cell disease by inhibiting red blood cell dehydration thus increasing hemoglobin. Senicapoc showed promising efficacy both in mice and humans (up to phase 3 study), with no adverse effects compared to placebo. Yet, the trial was terminated before end of study, as it seemed impossible to reach the primary endpoint [35]. Additionally, clinical studies using 
Clotrimazole, a small molecule inhibitor of $\mathrm{K}_{\mathrm{Ca}} 3.1$, in rheumatoid arthritis were performed in the 1980's [20]. Clotrimazole was shown to be effective in the treatment of the disease and superior to ketoprofen in certain measurements. Improvement with clotrimazole took place more slowly but was more sustained than with ketoprofen. However, further exploration of Cloritmazol's clinical utility was hampered by its gastrointestinal side effects and liver toxicity due to inhibition of cytochrome P450 [36].

ENV-59-GP3 is isolated from an endogenous retrovirus. Endogenous retroviruses originate from ancient viral infections. Evolution of the interaction between ion channels and viral envelope proteins might originally have been a result of the optimization of the ENV structure for its primary use i.e. viral entry. It is feasible that ion channels might provide additional attachment points for the virus to target cells and thus function as co-receptors. Alternatively, changes in the membrane potential might play a role in viral entry into the target cells. It is feasible that the ISD sequences from exogenous or other families of endogenous retroviral envelopes are also inhibitors of the $\mathrm{K}_{\mathrm{Ca}} 3.1$ or other similar ion channels. Indeed, it is possible that other virus groups or other pathogens have evolved similar immune modulatory activities independently. Whether this is the case remains to be investigated.

In any case, the data presented here has important implications for our understanding of endogenous retroviruses in immunology and the relationship between the ENV59 peptide and IBD disease provides further rationale for $\mathrm{K}_{\mathrm{Ca}} 3.1$ constituting an attractive drug target.

\section{Figure legends}

Figure 1 Effect of the ENV59-GP3 peptide and $\mathrm{K}_{\mathrm{Ca}} 3.1$-blocker NS6180 in DSS-induced acute colitis mice model .

Either saline (control) or ENV59-GP3 peptide $(0.5 \mathrm{mg} / \mathrm{mg})$ or NS6180 blocker $(2.5 \mathrm{mg} / \mathrm{kg})$ were administrated intraperitoneally every other day of DSS treatment. Weight development was monitored from day 0 to day 10. (A) daily weight development is shown for each of the treatment groups (pool of 2 independent experiments, 9-10 mice in each group). (B) The comparison of colon length. Data are expressed as means \pm SEM ( $\mathrm{n}=10$ per group). ${ }^{*} \mathrm{p}<0.05, * * * \mathrm{p}<0.001$, and $* * * * \mathrm{p}<0.0001$, one-way ANOVA with post hoc Tukey's test. (C) Evaluation of inflammatory cytokines. Total distal colon tissue was isolated from mice 10 days after the induction of DSS colitis and relevant treatments and equivalent biopsy sections were taken for inflammatory mRNA analysis (RT-qPCR). All gene values were normalized to the expression of Hprt1. Data are presented as mean $\pm \operatorname{SEM}(\mathrm{n}=10$ per group).

Figure 2 Histomorphology of colon tissue and inflammation scores according to the scale described in the Materials and Methods. (A) Inflammation score for each treatment group. The histological H\&E stains were scored by 2 individuals blinded to treatment and experiments. Right panel, Representative H\&E staining from mouse colon. Bar $=100 \mu \mathrm{m}$. Differences were calculated using a one-way analysis of variance (ANOVA) followed by post hoc Tukey's test test. Values are presented as mean $\pm \mathrm{SD} .{ }^{* *} P<0.01,{ }^{* * *} P$ $<0.001$.

Figure 3 A) Functional expression of $\mathrm{K}_{\mathrm{Ca}} 3.1$ and $\mathrm{Kv}$ channels in THP-1 cells. Application of NS309 or $1 \mu \mathrm{M}$ NS6180 occurred by switching to the appropriate tube feeding the inflow-line. From left to the right: The IV curve recorded after stabilization of the current after the initial run up (Control) and 1 min after application of $500 \mathrm{nM}$ NS309.: After NS309 wash-out and establishment of new stable baseline (NS309 wash-out) $1 \mu \mathrm{M}$ NS6180 was applied (NS6180) which completely blocked the KCa3.1 component. Note the distinct depolarizing shift in reversal potential. : The difference curve obtained by subtracting the curves in the middle panel revealing the NS6180 sensitive component. B) Expression of KCNN4 mRNA in THP-1 cells. Melting curve plot showing Q-PCR product specific for KCNN4 mRNA. C) Cytokine release and inhibiting effect of Env59-GP3 and of NS6180 in stimulated THP-1 cells.mRNA and protein levels for IL-6 and TNF-alpha.

Figure 4 A) Calcium influx following Ca 2+ depletion in THP-1 cells. Calcium flux to the cytosol following cell activation is reduced in the presence of Env59-GP3 or NS6180. B-C) Selective inhibition 
of THP-1 $\mathrm{K}_{\mathrm{Ca}} 3.1$ channels by Env59-GP3. Whole cell current recording from THP-1 cell. B shows the IV curves after current stabilization for about $15 \mathrm{~min}$ (a) and after perfusion with Env59-GP3 peptide at a concentration of $20 \mu \mathrm{M}$ for $5 \mathrm{~min}$ (b) (left figure). The IV curve after Env59-GP3 peptide wash-out (c ) (right figure) and after perfusion with $1 \mu \mathrm{M}$ NS6180 for $5 \mathrm{~min}(\mathrm{~d})$. The arrows indicate $-40 \mathrm{mV}$, the membrane potential used for putative "pure" $\mathrm{K}_{\mathrm{Ca}} 3.1$ current recording. The $-40 \mathrm{mM}$ current recordings were used for construction of the experimental time course relation shown in $\mathrm{C}$, where the letters mark the times for the trace recordings. D-E) $\mathrm{IC}_{50}$ value for ENV59-GP3 in recombinant human $\mathrm{K}_{\mathbf{C a}} 3.1$ channels stably expressed in HEK293 cells. The fraction of unblocked current at the end of compound application as a function of the test concentration. The lines are the curves resulting from fits of the data to the Hill equation described by IC50 values of $43 \mu \mathrm{M}$ for Env59-GP3 peptide.

Figure 5 A-D. ENV59-GP3 inhibition of recombinant human $\mathrm{K}_{\mathrm{Ca}} 3.1$ channels stably expressed in HEK293 cells . A,B,C show the $\mathrm{K}_{\mathrm{Ca}} 3.1$ currents in the absence (a,c.e) and presence (b,d,f) of compounds, with the letters also showing the times of recording at the time course in D. After establishment of the whole cell configuration $\mathrm{K}_{\mathrm{Ca}} 3.1$ currents increased as expected due to equilibration with the buffered Ca2+ solution in the pipette (compare also to Fig. 4) and then ran down to a stable current (D). The reversible $\mathrm{K}_{\mathrm{Ca}} 3.1$ blocking scorpion toxin peptide Charybdotoxin $(10 \mathrm{nM})(\mathrm{A}, \mathrm{D})$ and the $\mathrm{K}_{\mathrm{Ca}} 3.1$ positive modulator NS309 $(500 \mathrm{nM})(\mathrm{D})$ framed two additions of ENV59-GP3 $(50 \mu \mathrm{M})(\mathrm{B}, \mathrm{D})$ and the control peptide Flu ISU F\#2 $(50 \mu \mathrm{M})(\mathrm{C}, \mathrm{D})$, respectively.

\section{References:}

1. Kaser, A., S. Zeissig, and R.S. Blumberg, Genes and Environment: How Will Our Concepts on the Pathophysiology of IBD Develop in the Future? Digestive Diseases, 2010. 28 (3): p. 395-405.

2. Limbergen, J.V., D.C. Wilson, and J. Satsangi, The Genetics of Crohn's Disease. 2009. 10 (1): p. 89-116.

3. Ananthakrishnan, A.N., Environmental risk factors for inflammatory bowel disease. Gastroenterology \& hepatology, 2013.9 (6): p. 367-374.

4. Eichele, D.D. and K.K. Kharbanda, Dextran sodium sulfate colitis murine model: An indispensable tool for advancing our understanding of inflammatory bowel diseases pathogenesis. World journal of gastroenterology, 2017. 23 (33): p. 6016-6029.

5. Kiesler, P., I.J. Fuss, and W. Strober, Experimental Models of Inflammatory Bowel Diseases. Cell Mol Gastroenterol Hepatol, 2015.1 (2): p. 154-170.

6. Chassaing, B., et al., Dextran sulfate sodium (DSS)-induced colitis in mice. Curr Protoc Immunol, 2014. 104 : p. 15.25.1-15.25.14.

7. Perše, M. and A. Cerar, Dextran Sodium Sulphate Colitis Mouse Model: Traps and Tricks. Journal of Biomedicine and Biotechnology, 2012. 2012 : p. 718617.

8. Yan, Y., et al., Temporal and spatial analysis of clinical and molecular parameters in dextran sodium sulfate induced colitis. PLoS One, 2009. 4 (6): p. e6073.

9. Laska, M.J., et al., Human Endogenous Retroviral Genetic Element With Immunosuppressive Activity in Both Human Autoimmune Diseases and Experimental Arthritis. Arthritis Rheumatol, 2017.69 (2): p. 398-409.

10. Bahrami, S., et al., Immunomodulating peptides derived from different human endogenous retroviruses (HERVs) show dissimilar impact on pathogenesis of a multiple sclerosis animal disease model. Clin Immunol, 2018. 191 : p. 37-43.

11. Di, L., et al., Inhibition of the $K<$ sup $>+</$ sup $>$ channel KCa3.1 ameliorates $T$ cell-mediated colitis. 2010. 107 (4): p. 1541-1546. 
12. Hansen, L.K., The role of T cell potassium channels, KV1.3 and KCa3.1, in the inflammatory cascade in ulcerative colitis. Dan Med J, 2014. 61 (11): p. B4946.

13. Castle, N.A., Therapeutic potential of KCa3.1 blockers: recent advances and promising trends $A U$ Wulff, Heike. Expert Review of Clinical Pharmacology, 2010. 3 (3): p. 385-396.

14. Begenisich, T., et al., Physiological roles of the intermediate conductance, Ca2+-activated potassium channel Kcnn4. J Biol Chem, 2004. 279 (46): p. 47681-7.

15. Cahalan, M.D. and K.G. Chandy, Ion channels in the immune system as targets for immunosuppression. Curr Opin Biotechnol, 1997.8 (6): p. 749-56.

16. Cahalan, M.D. and K.G. Chandy, The functional network of ion channels in T lymphocytes. Immunological reviews, 2009.231 (1): p. 59-87.

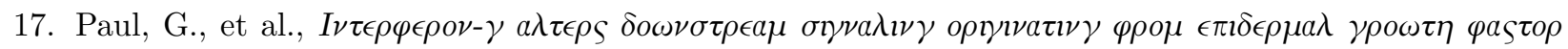

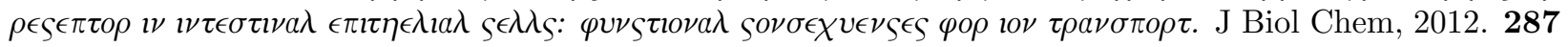
(3): p. $2144-55$.

18. Strøbæk, D., et al., NS6180, a new K(Ca) 3.1 channel inhibitor prevents T-cell activation and inflammation in a rat model of inflammatory bowel disease. British journal of pharmacology, 2013.168 (2): p. 432-444.

19. Reich, E.P., et al., Blocking ion channel KCNN4 alleviates the symptoms of experimental autoimmune encephalomyelitis in mice. Eur J Immunol, 2005. 35 (4): p. 1027-36.

20. Wojtulewski, J.A., et al., Clotrimazole in rheumatoid arthritis. Annals of the rheumatic diseases, 1980. 39 (5): p. 469-472.

21. Holm, C.K., et al., Influenza A virus targets a cGAS-independent STING pathway that controls enveloped RNA viruses. Nat Commun, 2016. 7 : p. 10680.

22. Nguyen, H.T., et al., CD98 expression modulates intestinal homeostasis, inflammation, and colitisassociated cancer in mice. J Clin Invest, 2011. 121 (5): p. 1733-47.

23. Viennois, E., et al., Dextran sodium sulfate inhibits the activities of both polymerase and reverse transcriptase: lithium chloride purification, a rapid and efficient technique to purify RNA.BMC Res Notes, 2013. 6 : p. 360.

24. Sankaranarayanan, A., et al.,Naphtho[1,2-<em>d</em>]thiazol-2-ylamine (SKA-31), a New Activator of KCa2 and KCa3.1 Potassium Channels, Potentiates the Endothelium-Derived Hyperpolarizing Factor Response and Lowers Blood Pressure. 2009. 75 (2): p. 281-295.

25. Lytton, J., M. Westlin, and M.R. Hanley, Thapsigargin inhibits the sarcoplasmic or endoplasmic reticulum Ca-ATPase family of calcium pumps. J Biol Chem, 1991. 266 (26): p. 17067-71.

26. Chen, R. and S.H. Chung, Molecular dynamics simulations of scorpion toxin recognition by the Ca(2+)activated potassium channel KCa3.1. Biophys J, 2013. 105 (8): p. 1829-37.

27. Lam, J. and H. Wulff, The Lymphocyte Potassium Channels Kv1.3 and KCa3.1 as Targets for Immunosuppression. Drug development research, 2011. 72 (7): p. 573-584.

28. Chou, C.C., C.A. Lunn, and N.J. Murgolo, KCa3.1: target and marker for cancer, autoimmune disorder and vascular inflammation? Expert Rev Mol Diagn, 2008. 8 (2): p. 179-87.

29. Ohya, S. and H. Kito, Ca(2+)-Activated $K(+)$ Channel KCa3.1 as a Therapeutic Target for Immune Disorders. Biol Pharm Bull, 2018.41 (8): p. 1158-1163.

30. O'Connor, W., Jr., et al., A protective function for interleukin 17 A in T cell-mediated intestinal inflammation. Nat Immunol, 2009. 10 (6): p. 603-9. 
31. Hansen, L.K., et al., Expression of T-cell KV1.3 potassium channel correlates with pro-inflammatory cytokines and disease activity in ulcerative colitis. Journal of Crohn's and Colitis, 2014.8 (11): p. 1378-1391.

32. Simms, L.A., et al., KCNN4Gene Variant Is Associated With Ileal Crohn's Disease in the Australian and New Zealand Population.2010. 105 (10): p. 2209-2217.

33. Yu, Z.H., et al., Targeted inhibition of KCa3.1 channel attenuates airway inflammation and remodeling in allergic asthma. Am J Respir Cell Mol Biol, 2013. 48 (6): p. 685-93.

34. Chen, Y.J., et al., The KCa3.1 blocker TRAM-34 reduces infarction and neurological deficit in a rat model of ischemia/reperfusion stroke. J Cereb Blood Flow Metab, 2011.31 (12): p. 2363-74.

35. Ataga, K.I., et al., Efficacy and safety of the Gardos channel blocker, senicapoc (ICA-17043), in patients with sickle cell anemia.Blood, 2008. 111 (8): p. 3991-7.

36. Alvarez, J., M. Montero, and J. Garcia-Sancho, High affinity inhibition of Ca(2+)-dependent K+ channels by cytochrome P-450 inhibitors. J Biol Chem, 1992. 267 (17): p. 11789-93.
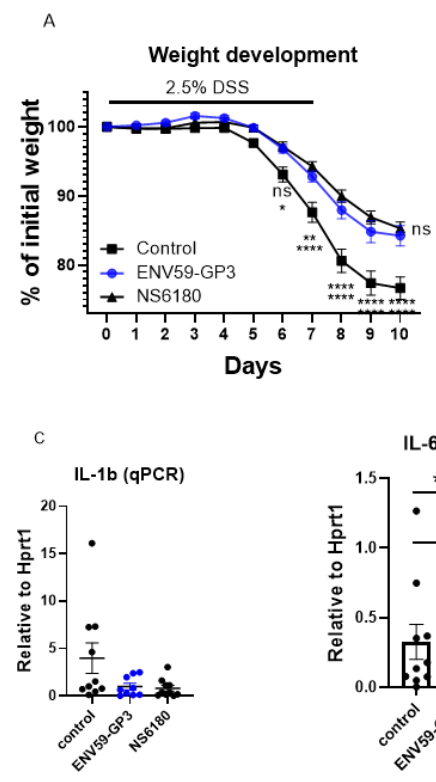

A

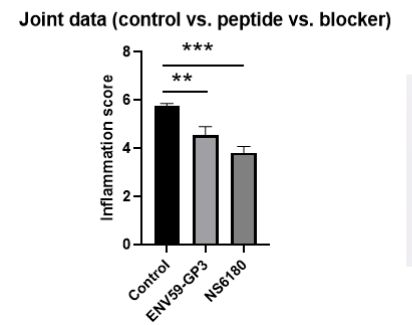

B

Colon length day 10
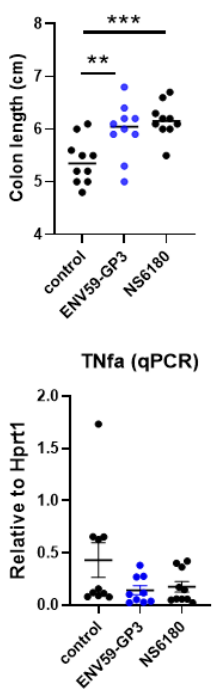

ENV59-GP3

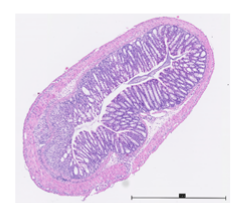

NS6180

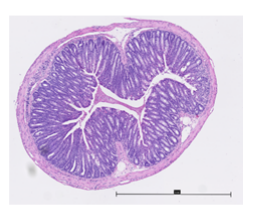


A

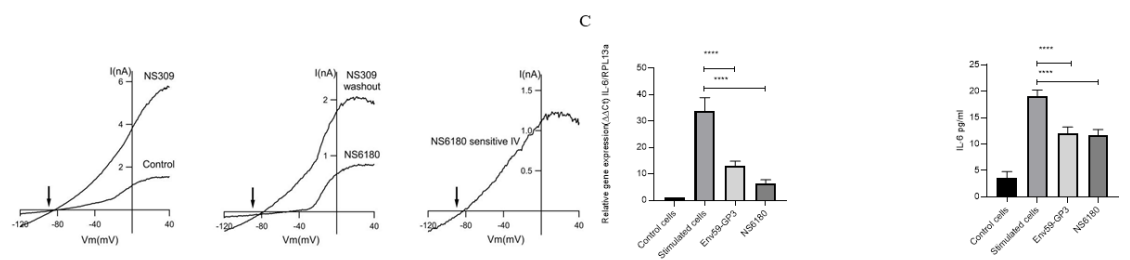

B
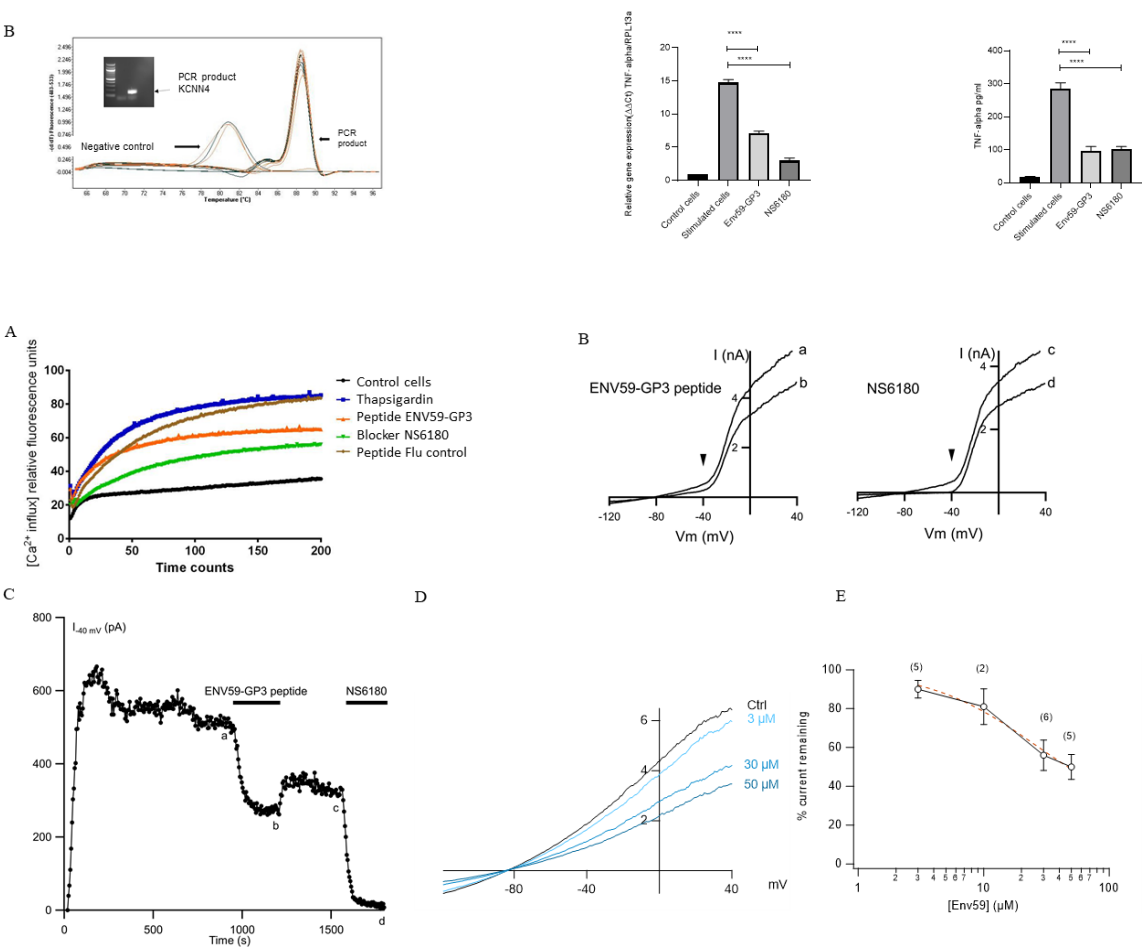

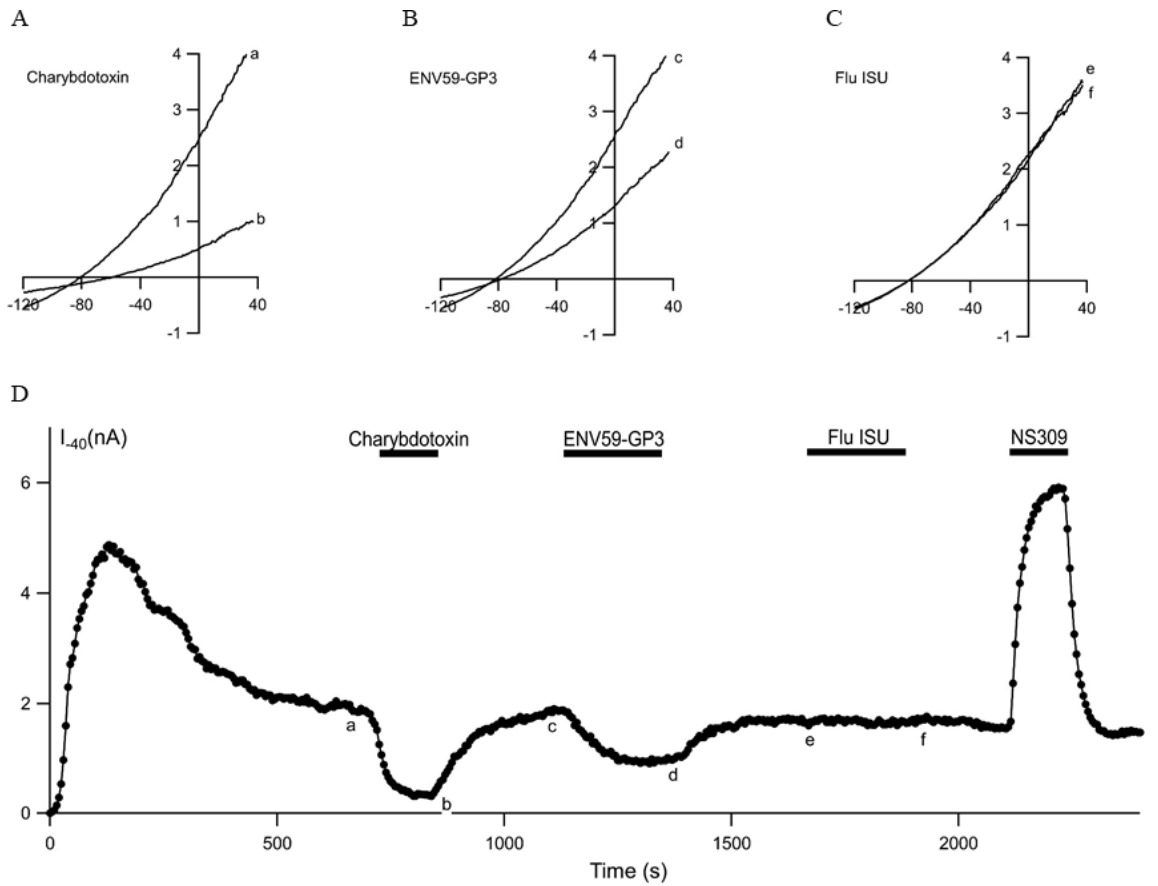